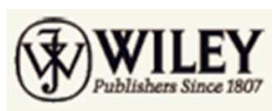

\title{
Quantitative analysis of tibial subchondral bone: texture analysis outperforms conventional trabecular microarchitecture analysis
}

\begin{tabular}{|r|l|}
\hline Journal: & Journal of Magnetic Resonance Imaging \\
\hline Manuscript ID: & Draft \\
\hline Wiley - Manuscript type: & Original Research \\
\hline Classification: & $\begin{array}{l}\text { Bone < Musculoskeletal imaging < Clinical Science, Joint (knee, shoulder, } \\
\text { wrist) < Musculoskeletal imaging < Clinical Science, Imaging techniques } \\
\text { and processing < Imaging technology and safety < Basic Science }\end{array}$ \\
\hline Manuscript Keywords: & osteoarthritis, subchondral bone, MRI, texture analysis \\
\hline
\end{tabular}

SCHOLARONE

Manuscripts 
QUANTITATIVE ANALYSIS OF TIBIAL SUBCHONDRAL BONE: TEXTURE ANALYSIS OUTPERFORMS CONVENTIONAL TRABECULAR

MICROARCHITECTURE ANALYSIS 


\begin{abstract}
PURPOSE

To compare two different methods of quantitative assessment of tibial subchondral bone in osteoarthritis (OA): statistical texture analysis (sTA) and trabecular microarchitecture analysis (tMA).

METHODS
\end{abstract}

Asymptomatic controls aged 20-30 ( $n=10)$, patients aged 40-50 with chronic knee pain but without established OA ( $n=10)$ and patients aged 55-85 with advanced OA scheduled for knee replacement $(n=10)$ underwent knee MR imaging at 3T with a 3D gradient echo (GRE) sequence to allow sTA and tMA.

tMA and sTA features were calculated using ROI creation in the medial (MT) and lateral (LT) tibial subchondral bone. Features were compared between groups using one-way ANOVA. The two most discriminating tMA and sTA features were used to construct exploratory discriminant functions to assess the ability of the two methods to classify participants.

RESULTS

No tMA features were significantly different between groups at either MT or LT. 17/20 and 11/20 sTA features were significantly different between groups at the MT/LT respectively $(p<0.001)$

Discriminant functions created using tMA features classified 12/30 participants correctly (40\% accuracy, 95\% Cl 22-58\%) based on MT data and 9/30 correctly $(30 \%, 14-46)$ based on 
LT data. Discriminant functions using sTA features classified 16/30 participants correctly

(53\%, 35-71) based on MT data and 14/30 correctly (47\%, 29-65) based on LT data.

\section{CONCLUSION}

sTA features showed more significant differences between the three study groups and improved classification accuracy compared to tMA features. 


\section{INTRODUCTION}

Osteoarthritis $(\mathrm{OA})$ is a whole joint disease affecting a number of different tissues including the subchondral bone (SB). The SB is usually defined as the combination of the SB plate, which separates articular cartilage from marrow cavity, together with adjacent subarticular trabeculae and marrow cavity(1).

The SB is believed to play an important role in OA pathogenesis via a number of mechanisms. There is biochemical cross-talk between SB and articular cartilage, and it has been suggested that various small molecules generated in the SB by osteoblasts during SB remodelling may predispose to overlying cartilage degradation(2). Breaching of the osteochondral junction by neurovascular bundles originating from the SB and resultant innervation of the usually aneural articular cartilage may result in the debilitating pain experienced by many OA sufferers(3). Changes in SB with OA progression are non-linear with an initial increase in bone remodelling with associated loss of bone density, followed by thickening of the SB plate in more advanced disease which eventually becomes manifest radiographically as subchondral sclerosis(4).

SB is a potential therapeutic target for OA given that changes have been demonstrated very early in disease before irreversible joint damage has occurred(5), and dynamic changes have been demonstrated in response to therapy(6). In order to develop and assess new treatments, reliable and accurate quantification of SB is required.

Various radiological techniques have been employed to provide quantitative analysis of SB. Dual-energy $\mathrm{x}$-ray absorptiometry (DXA) has been used to assess SB density which has suggested a protective effect of increased SB density on OA progression(7). However, 
substantial methodological variation between different studies limits generalizability of results(8).

Fractal signature analysis (FSA) assesses the self-similarity of an image at different magnifications. Conceptually it is an assessment of the degree of SB organisation or disorganisation(9). It has been performed using plain radiographs (XR), computed tomography $(C T)$ and magnetic resonance (MR) imaging. Differences in the XR fractal signatures of normal and osteoarthritic SB have been detected and it has been suggested that MR fractal signature may be able to detect early SB adaptation to altered loading conditions which precede established OA(10). However, FSA does appear sensitive to ROI placement and choice of fractal dimension which again may limit generalizability(11).

Trabecular microarchitecture analysis (tMA) involves calculating structural parameters analogous to those used in conventional histomorphometry including bone volume fraction (BV/TV), trabecular number (Tb.N), trabecular spacing (Tb.Sp) and trabecular thickness (Tb.Th)(12). The gold standard technique is microCT, however this is only able to image ex vivo specimens with scan durations lasting up to several hours(13). Nevertheless, tMA has proved feasible in vivo using high spatial resolution CT and MR. Of these, MR offers the advantage of slightly better prediction of ground-truth structural parameters as well as improved contrast resolution of other structures involved in the OA disease process such as articular cartilage and synovium(14, 15). Changes in MR tMA parameters have been demonstrated in OA subjects compared to normal controls, and there is good correlation with gold standard microCT(16). However, tMA relies on segmentation of the MR image into bone and marrow voxels. This requires setting of a threshold signal intensity value. Variations in this threshold can cause large variations in the calculated structural 
parameters(17). Moreover, the thin slices required to depict individual trabeculae and the gradient echo based sequences used to achieve the required spatial resolution in vivo can lead to reduced SNR, further hindering segmentation(15).

Statistical texture analysis (sTA) quantifies image texture based on the distribution of greylevel intensities within the image. It includes different classes of parameters, such as parameters based on the histogram of pixel values (corresponding to signal intensity when MR is analysed), parameters based on the spatial variation of pixel values across the image (absolute gradient class), parameters based on the number of adjacent pixels having the same intensity value (run length matrix class) and parameters based on the distribution of pairs of pixels (grey-level co-occurrence matrix class)(18). The value of sTA in medical imaging lies in its ability to detect subtle alterations in the imaging characteristics of a tissue before they are visible to the naked eye.

sTA has been used for a variety of applications in the musculoskeletal system(19-21). sTA of tibial SB using low field strength MR (0.18 T) was able to predict which knees would develop rapidly progressive cartilage loss in a longitudinal study(22). Differences in the histogram variance of tibial SB have been demonstrated in subjects with possible early $\mathrm{OA}(23)$

sTA offers the potential advantages over alternative methods of SB quantification, such as tMA, of not requiring segmentation and allowing for increased slice thickness (and hence increased SNR). Previous studies have demonstrated good reliability across different MR platforms and between observers, and correlation with histomorphometry(24-26). However, it remains unclear which method of SB quantification is optimal. Answering this 
question would be useful to determine the preferred method for use in future studies of SB in OA.

The aim of this study was to compare two different methods of quantitative assessment of tibial SB in OA: sTA and tMA. 


\section{METHODS}

Ethical approval for the study was obtained from the Local Research Ethics Committee. All subjects provided written, informed consent. This was a prospective, observational study, carried out at our institution between February and August 2014.

\section{Participants}

Three groups of 10 participants were recruited. Group 1 contained 10 asymptomatic volunteers aged 20-30 who had a normal BMI (body mass index). Group 2 contained 10 participants aged between 40-50 who had been referred to the Orthopaedic service at our institution with chronic non-traumatic knee pain, and had knee radiographs demonstrating no significant OA (Kellgren-Lawrence grade < 2)(27). Group 3 contained 10 participants aged 55-85 with advanced OA who were scheduled to undergo total knee replacement (TKR).

These participant groups were designed to provide a cross-sectional sample of various stages of OA, including normal/no OA (group 1), at risk of OA/possible early OA (group 2) and advanced OA (group 3). In particular, we included individuals without radiographic evidence of $O A$ (rather than individuals with established radiographic mild $O A$ ) in group 2 as this group with possible very early disease are the most likely to benefit from targeted preventative therapy, before established structural changes have occurred. Therefore it is of interest to be able to identify such individuals. The difficulties in identifying very early OA cohorts are well-documented, however the presence of chronic knee pain in middle aged individuals (such as group 2 in this study) is considered the most significant predictor of incident knee $\mathrm{OA}(28)$. 
Patients were excluded if there was a history of significant lower limb injury or lower limb surgery, inflammatory arthritis, haematological malignancy, bone metastases, metabolic bone disease or if there was a contraindication to MR imaging.

All participants had their height and weight recorded at the time of their MR examination and completed an Oxford Knee Score questionnaire to assess severity of symptoms(29).

Radiographs

All individuals in groups $2 \& 3$ underwent weight-bearing AP and lateral radiographs of the symptomatic knee. The Kellgren-Lawrence grade was assessed by two observers $(* *$ BLINDED**), both Radiology residents with 3 years' experience, with disagreements resolved by consensus with a senior reader (**BLINDED**), a musculoskeletal radiologist with 12 years' experience. Individuals in group 2 were excluded if there was evidence of OA (Kellgren-Lawrence grade $\geq 2$ ).

\section{MR Acquisition}

Individuals underwent MR imaging of the symptomatic knee (in group 1 individuals a randomly selected knee was used) on a wide-bore 3T MR platform (GE Healthcare, Amersham, UK) using an 8 channel high definition knee coil (GE WD 750).

The MR protocol featured a sagittal intermediate-weighted, fat-saturated spin echo sequence (FOV $15 \times 15.4$ cm, matrix $352 \times 288$, TR $3422 \mathrm{mSec}$, TE $48.31 \mathrm{mSec}$, number of excitations (NEX) 1, slice thickness $3 \mathrm{~mm}$, interslice gap $4 \mathrm{~mm}$, scan duration 4 mins 30 seconds) to evaluate for the presence of bone marrow lesions (BML) or focal cartilage defects. 
To evaluate the SB, we performed a coronal 3D gradient echo (GRE) sequence (FOV $12 \mathrm{x}$ $12.3 \mathrm{~cm}$, matrix $512 \times 512$, TR 11.58 , flip angle $50^{\circ}$, TE 4.33, NEX 0.6, slice thickness $1 \mathrm{~mm}$, in-plane spatial resolution $0.23 \times 0.24 \mathrm{~mm}$, scan duration 3 mins 45 seconds) through the central weight bearing tibial plateau (figure 1).

\section{Clinical MR Analysis}

All MR studies were reviewed by a consultant musculoskeletal radiologist with 12 years' experience (AT). As the purpose of group 2 was to include individuals with possible early OA, any participants in group 2 with MR evidence of established OA - full thickness cartilage defects or BMLs - were excluded. One potential group 2 subject was excluded due to a full thickness cartilage defect. The MR studies of group 1 participants were also reviewed to ensure that there was no structural abnormality.

\section{Trabecular microarchitecture}

tMA was performed using the BoneJ plugin for ImageJ (National Institutes of Health, Bethesda, Maryland)(30). Twenty 3D GRE images (representing a $20 \mathrm{~mm}$ thick volume) through the central weight bearing portion of the tibial plateau were selected via reference to axial and coronal localizers. Rectangular regions of interest (ROI) measuring $5.4 \mathrm{~mm}$ in height $\times 20.9 \mathrm{~mm}$ in width were created in the medial (MT) and lateral (LT) tibial SB on each slice. This ROI size was chosen following pilot testing to enable accurate ROI placement across a variety of different tibial plateau contours and widths. ROI placement was as close as possible to the osteochondral junction at the superior aspect (figure 1). Care was taken to avoid the cortical bone at the medial/lateral borders of the tibial plateau. 
Bone/marrow segmentation was performed using a previously described dual thresholding technique based on estimating the reference intensity levels of bone and marrow on the signal intensity histogram of a background ROI placed in the femoral trabecular bone (31). Standard algorithms were then used to calculate apparent 2D trabecular microarchitecture properties analogous to those used for histomorphometric analysis: apparent trabecular bone volume fraction (aBV/TV), apparent trabecular thickness (aTb.Th), apparent trabecular spacing (aTb.Sp) and apparent trabecular number (aTb.N). Detailed descriptions of the method of calculating these properties have been provided previously $(17,32)$. In brief, aBV/TV is the percentage of the number of bone pixels divided by the total number of pixels in the ROI, aTb. Th is calculated using an algorithm defining trabecular thickness as the diameter of the greatest circle that fits within a given trabeculum, aTb.Sp is the thickness of the background marrow calculated using a similar method, and aTb.N is the aBV/TV divided by aTb.Th. For statistical analysis, tMA properties were averaged across all 20 slices to give a summary measure for each participant. Analyses were performed separately for both MT and LT SB.

\section{Statistical texture analysis}

sTA was performed on the medial and lateral tibial SB using dedicated software (MazDa version 4.6)(33). Six GRE coronal images through the central weight-bearing portion of the tibial plateau (determined by cross referencing to sagittal and axial localizers and corresponding to the volume used for tMA) were selected for each subject. Regions of interest (ROI) of identical size and position to those used for tMA were created in the MT and LT SB on each slice (figure 1). 
Twenty texture features (tables $2 \& 3$ ) were extracted for each region of interest on each slice. A more detailed description of the texture parameters calculated has been provided previously $(34,35)$. Run-length matrix (RLM) parameters are calculated 4 times for each ROI (in vertical, horizontal, $45^{\circ}, 135^{\circ}$ directions) and grey-level co-occurrence matrix (GLCM) parameters are calculated 20 times for each ROI at a variety of pixel offsets. For statistical analysis, the mean value of RLM and GLCM parameters was used for each ROI, giving a total of 20 parameters to be analysed. Each of the 20 TA parameters was then averaged across all 6 slices to give a summary measure for each participant. Analyses were performed separately for both MT and LT SB.

Sample size

In the absence of any reliable pilot data for the current study, we selected a sample size of 30 as being similar to previous feasibility studies evaluating novel methods of assessing $\mathrm{SB}(36,37)$.

Statistics

Following visual assessment for a normal distribution using Q-Q plots, mean tMA and sTA features were compared between groups using ANOVA, using the Bonferroni method to correct for multiplicity of testing. For tMA features $(n=4)$, a p value of $<0.0125(0.05 / 4)$ was considered significant. For sTA features $(n=20)$, a p value of $<0.0025(0.05 / 20)$ was considered significant. Where a feature demonstrated significant differences between all three groups, post-hoc unpaired Student's $t$-tests were performed to evaluate for significant differences between the individual groups, with significance levels as above. 
To assess the ability of tMA and sTA features to classify participants into the correct group, the two tMA and two sTA features which were best able to discriminate between groups were selected by calculating the Fisher coefficient (the ratio of between-group variance to within-group variance). The selected features were then used to create two exploratory linear (canonical) discriminant functions using tMA and sTA features respectively. The classification accuracy of each function was then assessed using discriminant analysis with leave-one-out cross-validation, expressed as percentage accuracy.

Region of interest creation for both tMA and STA was performed by two independent observers, a subset of three Radiology residents with 3 years' experience (**BLINDED**) and 1 year's experience (**BLINDED**). **BLINDED** and **BLINDED** performed tMA,$* *$ BLINDED** and **BLINDED** performed sTA. Reproducibility was assessed by calculating coefficients of variation (CV) and intraclass correlation coefficients (ICC - single measures, absolute agreement) for each tMA and sTA feature. 


\author{
RESULTS \\ Participant characteristics \\ Participant characteristics are summarized in table 1. \\ Between-group comparisons
}

Results are summarized in tables 2 and 3 for MT and LT SB respectively.

At the MT, no mean tMA parameters (0/4) were significantly different between groups.

Seventeen out of 20 mean sTA parameters were significantly different between groups. In post-hoc $t$-tests, no sTA parameters were significantly different between groups 1 and 2 , 14 sTA parameters were significantly different between groups 2 and 3, and 14 sTA parameters were significantly different between groups 1 and 3. Percentage differences between groups ranged from $0-26.1 \%$ for $\mathrm{tMA}$ parameters, with the greatest differences in aTb.Th and $0-176.5 \%$ for sTA parameters, with the greatest differences in gradient kurtosis (figure 2).

At the LT, no mean tMA parameters (0/4) were significantly different between groups. Eleven out of 20 mean sTA parameters were significantly different between groups. In post-hoc t-tests, no sTA parameters were significantly different between groups 1 and 2, three sTA parameters were significantly different between groups 2 and 3 , and 11 sTA parameters were significantly different between groups 1 and 3. Percentage differences between groups ranged from $0-27.3 \%$ for tMA parameters, with the greatest differences in $\mathrm{ABV} / \mathrm{TV}$ and $0-112.5 \%$ for sTA parameters, with the greatest differences in histogram kurtosis. 


\section{Classification}

Results are summarized in table 4.

The best classification results at both the MT and LT were obtained using sTA parameters.

At the MT a discriminant function using the number of pixels with non-zero gradient (absolute gradient class) and inverse different moment (grey-level co-occurrence matrix class) classified $16 / 30$ subjects correctly (53\%, 95\% CI 35-71\%). Using tMA parameters (aBV/TV and aTb.Th), 13/30 subjects were classified correctly (43.3\%, 25-61\%)

At the $L T$, a discriminant function using the sTA parameters histogram mean and histogram variance classified $14 / 30$ subjects correctly (47\%, 25-61\%). Using tMA parameters (aBV/TV and aTb.Sp), 9/30 subjects were classified correctly (30\%, 14-46\%).

\section{Reliability}

Results are summarized in table 5 .

Fourteen out of 20 sTA parameters had ICC values $>0.81$ indicating near-perfect interobserver reliability, 4/20 parameters had ICCs between $0.61-0.81$ indicating good reliability, and 2 parameters had ICCs between $0.41-0.61$ indicating moderate reliability, using standard interpretation of ICC values(38). CVs ranged from $0.2 \%$ (the RLM parameter short run-length emphasis) to $52.5 \%$ (histogram kurtosis). 13/20 sTA parameters had CVs of less than $10 \%$.

Two out of four tMA parameters (aBV/TV and aTb.N) had ICC values between 0.61-0.81 indicating good reliability, with the other two parameters (aTb.Th and aTb.Sp) having ICC 
values between $0.41-0.61$ indicating moderate reliability. CVs ranged from $11.7 \%$ (aTb.Sp) to $17.0 \%(a B V / T V)$.

1

2

3

4

5

6

7

8

9

10

11

12

13

14

15

16

17

18

19

20

21

22

23

24

25

26

27

28

29

30

31

32

33

34

35

36

37

38

39

40

41

42

43

44

45

46

47

48

49

50

51

52

53

54

55

56

57

58

59

60

Journal of Magnetic Resonance Imaging 


\section{DISCUSSION}

Multiple sTA parameters demonstrated significant differences between the three study groups, whereas no tMA parameters were significantly different. Greater percentage differences between groups were demonstrated with sTA parameters. Classification of subjects using sTA parameters was more accurate than classification using tMA parameters.

sTA offers a surrogate or indirect assessment of SB architecture as opposed to the direct structural assessment of tMA. Nevertheless, our results suggest that sTA may be better at detecting alterations in SB architecture in OA. We offer two potential explanations for this apparent superiority.

First, sTA may be better suited to bone analysis on relatively low resolution images. tMA calculates parameters analogous to those used in histomorphometry. This was developed as a method of quantitative assessment of trabecular bone obtained from bone biopsies, performed under the microscope(12). While microCT is able to provide similar resolution to histological analysis, clinically feasible MR imaging is not - although with the advent of MR platforms of higher field strength, this may change. With current technology, partial volume effects and susceptibility artefact at the bone marrow interface are significant with the magnitude of error approaching the magnitude of tMA measurements themselves(17). In contrast, sTA was developed as a method of analysing computerised images at a variety of spatial resolutions including lower resolution images(34). It could therefore be argued that STA is fundamentally a more suitable technique for analysing SB on current clinical MR images with relatively low spatial resolution. 
Second, sTA may be more sensitive to early SB architectural changes compared with tMA. Changes in STA parameters have been demonstrated prior to any discernible changes in morphology or structure in other parts of the body(24). Similar superiority of surrogate measurement over direct measurement of SB architecture has been demonstrated when analysing SB on knee radiographs where FSA outperformed DXA(39). Each sTA parameter measures a particular property of the arrangement of pixels within an ROI such as variance, contrast and branching. Alterations in pixel arrangement which may be sufficient to manifest as a significant change in STA parameters may not be sufficient in magnitude to cause a significant change in tMA parameters.

While sTA is a surrogate measure of SB architecture, conceptually a number of sTA parameters have correlates with trabecular changes that are known to occur in OA such as trabecular discontinuity, thickening and disorganisation(40). For example, an increase in tissue disorganisation is likely to manifest as increased heterogeneity within the ROI. This would be reflected by changes in histogram-based features such as variance, as has been demonstrated previously and again was seen in this study(23). Trabecular thickening with loss of the normal fine linear pattern would reduce the number of transitions within the ROI from areas of high signal to areas of low signal (and vice versa), causing changes in the absolute gradient class of parameter. Loss of trabecular continuity would reduce the number of pixels occurring in runs, manifesting as changes to RLM class parameters.

A greater number of studies evaluating SB in OA have utilised tMA compared to those utilitsing sTA. sTA is the older technique (first described in 1973) with the tMA parameters used in recent studies being originally described more than a decade later(12, 34). However, the development of sTA as a method of quantifying bone architecture has been a 
more recent development (c.2010)(41). The results of this study suggest that sTA may offer additional information to tMA in the quantification of SB in OA and challenge the conventional practice of using tMA alone for such evaluation.

The changes in SB architecture demonstrated between groups are in keeping with previous studies demonstrating changes in the MR appearance of SB in OA. Of particular interest are those parameters which demonstrated differences between groups 1 and 2. Although these differences were non-significant, it is likely that we were underpowered in this regard. Individuals in group 2 had no radiographic evidence of OA (Kellgren-Lawrence $<2$ ), no BML, and no focal cartilage defects. Therefore, the MR changes in the SB may reflect very early disease. This has potential clinical utility in terms of identifying patients with very early disease who may be suitable for targeted preventative therapy.

There was substantial variation in the inter-observer reliability of sTA parameters, however the majority demonstrated near-perfect reliability based on standard interpretation of the ICC values. The CVs were, in general, substantially lower than the measured differences in parameters between groups, suggesting that they have sufficient sensitivity for further studies in OA. tMA parameters demonstrated moderate to good reliability based on ICC values. The CVs of between $10-20 \%$ were closer to the magnitude of the measured differences in parameters between groups, possibly indicating lower sensitivity to SB alterations.

Limitations of this study included a lack of histological correlation for our SB analyses. However, both sTA and tMA have previously demonstrated good correlation with groundtruth histomorphometry analyses $(16,26)$. The generalizability of our results is limited by the cross-sectional design, and the fact that our MR images were obtained at a single time 
point and using a single MR platform. Calculated sTA and tMA parameters have been shown to vary across MR platforms, predominantly due to differing acquisition parameters $(15,42)$. However, while sTA parameter values are sensitive to changes in acquisition parameters, their ability to distinguish different tissue types remains(25). Further work is needed to determine whether the distinction between different stages of alteration in SB architecture demonstrated in this study is reproducible across different platforms and institutions.

In conclusion, sTA features showed more significant differences between the three study groups containing individuals at different stages of $\mathrm{OA}$, and improved classification accuracy compared to tMA features. 


\section{REFERENCES}

1. Madry H, Dijk CN, Mueller-Gerbl M: The basic science of the subchondral bone. Knee Surg Sports Traumatol Arthrosc 2010; 18:419-433.

2. Priam S, Bougault C, Houard $X$, et al.: Identification of soluble $14-3-3 \varepsilon$ as a novel subchondral bone mediator involved in cartilage degradation in osteoarthritis. Arthritis Rheum 2013; 65:1831-1842.

3. Suri S, Gill SE, de Camin SM, Wilson D, McWilliams DF, Walsh DA: Neurovascular invasion at the osteochondral junction and in osteophytes in osteoarthritis. Ann Rheum Dis 2007; 66:1423-1428.

4. Burr DB, Gallant MA: Bone remodelling in osteoarthritis. Nat Rev Rheumatol 2012; 8:665-673.

5. Anderson-MacKenzie JM, Quasnichka HL, Starr RL, Lewis EJ, Billingham MEJ, Bailey AJ: Fundamental subchondral bone changes in spontaneous knee osteoarthritis. Int J Biochem Cell Biol 2005; 37:224-236.

6. Multanen J, Nieminen MT, Häkkinen A, et al.: Effects of high-impact training on bone and articular cartilage: 12-month randomized controlled quantitative MRI study. J Bone Miner Res 2014; 29:192-201.

7. Cao Y, Stannus OP, Aitken D, et al.: Cross-sectional and longitudinal associations between systemic, subchondral bone mineral density and knee cartilage thickness in older adults with or without radiographic osteoarthritis. Ann Rheum Dis 2013. 
8. Sepriano A, Roman-Blas JA, Little RD, et al.: DXA in the assessment of subchondral bone mineral density in knee osteoarthritis-A semi-standardized protocol after systematic review. Semin Arthritis Rheum 2015.

9. Buckland-Wright JC, Lynch JA, Macfarlane DG: Fractal signature analysis measures cancellous bone organisation in macroradiographs of patients with knee osteoarthritis. Ann Rheum Dis 1996; 55:749-755.

10. Baum T, Sauerschnig M, Penzel J, et al.: Early changes of trabecular bone structure in asymptomatic subjects with knee malalignment. J Comput Assist Tomogr 2014; 38:137-141.

11. Kraus VB, Feng S, Wang S, et al.: Trabecular morphometry by fractal signature analysis is a novel marker of osteoarthritis progression. Arthritis Rheum 2009; 60:3711-3722.

12. Parfitt AM, Drezner MK, Glorieux FH, et al.: Bone histomorphometry: standardization of nomenclature, symbols, and units. Report of the ASBMR Histomorphometry Nomenclature Committee. J Bone Miner Res 1987; 2:595-610.

13. Thomsen JS, Laib A, Koller B, Prohaska S, Mosekilde L, Gowin W: Stereological measures of trabecular bone structure: comparison of 3D micro computed tomography with 2D histological sections in human proximal tibial bone biopsies. J Microsc 2005; 218(Pt 2):171-179.

14. Link TM, Majumdar S, Lin JC, et al.: Assessment of trabecular structure using high resolution CT images and texture analysis. J Comput Assist Tomogr 1998; 22:15-24.

15. Link TM, Vieth V, Stehling C, et al.: High-resolution MRI vs multislice spiral CT: which technique depicts the trabecular bone structure best? Eur Radiol 2003; 13:663-671. 
16. Driban JB, Barbe MF, Amin M, et al.: Validation of quantitative magnetic resonance imaging-based apparent bone volume fraction in peri-articular tibial bone of cadaveric knees. BMC Musculoskelet Disord 2014; 15:143.

17. Majumdar S, Newitt $D$, Jergas $M$, et al.: Evaluation of technical factors affecting the quantification of trabecular bone structure using magnetic resonance imaging. Bone 1995; $17: 417-430$.

18. Castellano G, Bonilha L, Li LM, Cendes F: Texture analysis of medical images. Clin Radiol 2004; 59:1061-1069.

19. Blumenkrantz G, Stahl R, Carballido-Gamio J, et al.: The feasibility of characterizing the spatial distribution of cartilage T2 using texture analysis. Osteoarthritis Cartilage 2008; 16:584-590.

20. Chuah TK, Van Reeth E, Sheah K, Poh CL: Texture analysis of bone marrow in knee MRI for classification of subjects with bone marrow lesion - data from the Osteoarthritis Initiative. Magn Reson Imaging 2013; 31:930-938.

21. Harrison LCV, Nikander R, Sikiö M, et al.: MRI texture analysis of femoral neck: Detection of exercise load-associated differences in trabecular bone. J Magn Reson Imaging $2011 ; 34: 1359-1366$.

22. Marques J, Genant HK, Lillholm M, Dam EB: Diagnosis of osteoarthritis and prognosis of tibial cartilage loss by quantification of tibia trabecular bone from MRI. Magn Reson Med Off J Soc Magn Reson Med Soc Magn Reson Med 2013; 70:568-575. 
23. MacKay JW, Godley KC, Toms AP: MRI signal-based quantification of subchondral bone at the tibial plateau: a population study. Skeletal Radiol 2014; 43:1567-1575.

24. Suoranta S, Holli-Helenius K, Koskenkorva P, et al.: 3D Texture Analysis Reveals Imperceptible MRI Textural Alterations in the Thalamus and Putamen in Progressive Myoclonic Epilepsy Type 1, EPM1. PLoS ONE 2013; 8:e69905.

25. Mayerhoefer ME, Breitenseher MJ, Kramer J, Aigner N, Hofmann S, Materka A: Texture analysis for tissue discrimination on T1-weighted MR images of the knee joint in a multicenter study: Transferability of texture features and comparison of feature selection methods and classifiers. J Magn Reson Imaging 2005; 22:674-680.

26. Tameem HZ, Selva LE, Sinha US: Texture measure from low resolution MR images to determine trabecular bone integrity in osteoporosis. In: Conference Proceedings of the Annual International Conference of the IEEE Engineering in Medicine and Biology Society, Lyon 2007:2027-2030.

27. Kellgren JH, Lawrence JS: Radiological assessment of osteo-arthrosis. Ann Rheum Dis 1957; 16:494-502.

28. Felson DT, Hodgson R: Identifying and Treating Preclinical and Early Osteoarthritis. Rheum Dis Clin N Am 2014; 40:699-710.

29. Dawson J, Fitzpatrick R, Murray D, Carr A: Questionnaire on the perceptions of patients about total knee replacement. J Bone Joint Surg Br 1998; 80-B:63-69.

30. Doube M, Kłosowski MM, Arganda-Carreras I, et al.: BoneJ: Free and extensible bone image analysis in ImageJ. Bone 2010; 47:1076-1079. 
31. Newitt DC, van Rietbergen B, Majumdar S: Processing and analysis of in vivo highresolution MR images of trabecular bone for longitudinal studies: reproducibility of structural measures and micro-finite element analysis derived mechanical properties. Osteoporos Int J 2002; 13:278-287.

32. Dougherty R, Kunzelmann K-H: Computing Local Thickness of 3D Structures with ImageJ. Microsc Microanal 2007; 13(Supplement S02):1678-1679.

33. Szczypiński PM, Strzelecki M, Materka A, Klepaczko A: MaZda--a software package for image texture analysis. Comput Methods Programs Biomed 2009; 94:66-76.

34. Haralick R, Shanmugam K, I Dinstein: Textural features for image classification. IEEE Trans Syst Man Cybern 1973; 3:610-621.

35. Tang X: Texture information in run-length matrices. IEEE Trans Image Process 1998; 7:1602-1609.

36. Bolbos RI, Zuo J, Banerjee S, et al.: Relationship between trabecular bone structure and articular cartilage morphology and relaxation times in early OA of the knee joint using parallel MRI at 3 T. Osteoarthritis Cartilage 2008; 16:1150-1159.

37. Chang G, Xia D, Chen C, et al.: 7T MRI detects deterioration in subchondral bone microarchitecture in subjects with mild knee osteoarthritis as compared with healthy controls. J Magn Reson Imaging 2014; 41:1311-1317.

38. Landis JR, Koch GG: The measurement of observer agreement for categorical data. Biometrics 1977; 33:159-174. 
39. Messent EA, Buckland-Wright JC, Blake GM: Fractal analysis of trabecular bone in knee osteoarthritis $(O A)$ is a more sensitive marker of disease status than bone mineral density (BMD). Calcif Tissue Int 2005; 76:419-425.

40. Bobinac D, Spanjol J, Zoricic S, Maric I: Changes in articular cartilage and subchondral bone histomorphometry in osteoarthritic knee joints in humans. Bone 2003; 32:284-290.

41. Ito M: Recent progress in bone imaging for osteoporosis research. J Bone Miner Metab $2011 ; 29: 131-140$.

42. Lerski RA, Schad LR, Luypaert R, et al.: Multicentre magnetic resonance texture analysis trial using reticulated foam test objects. Magn Reson Imaging 1999; 17:1025-1031. 
2

TABLES

TABLE 1. Baseline characteristics of study subjects

\begin{tabular}{llll}
\hline & Group 1 & Group 2 & Group 3 \\
\hline Age $^{\mathrm{a}}$ & $26.2(21-29)$ & $46.7(42-50)$ & $71.4(57-84)$ \\
Body mass index $\left(\mathrm{kg} / \mathrm{m}^{2}\right)^{\mathrm{a}}$ & $24.1(3.3)$ & $27.3(4.3)$ & $31.3(4.9)$ \\
Females/males & $4 / 6$ & $3 / 7$ & $7 / 3$ \\
Right knee/Left knee & $5 / 5$ & $7 / 3$ & $6 / 4$ \\
Oxford knee score $^{\mathrm{a}}$ & $48(0)$ & $28.1(8.3)$ & $17.4(4.4)$ \\
Kellgren-Lawrence grade 0/1/2/3/4 & $\mathrm{N} / \mathrm{A}$ & $4 / 6 / 0 / 0 / 0$ & $0 / 0 / 1 / 5 / 4$ \\
\hline
\end{tabular}

${ }^{a}$ values are mean (standard deviation) except age which is mean (range) 
TABLE 2. Results of between group comparisons at the medial tibial plateau. Parameters demonstrating significant differences between groups are highlighted in bold.

\begin{tabular}{|c|c|c|c|c|c|c|c|}
\hline Parameter & Group 1 * & Group 2 * & Group 3* & $\begin{array}{l}\mathrm{p} \text { (all } \\
\text { groups) }\end{array}$ & $\begin{array}{l}\text { \% } \\
\text { difference } \\
\text { (group 1/2) }\end{array}$ & $\begin{array}{l}\text { \% } \\
\text { difference } \\
\text { (group 2/3) }\end{array}$ & $\begin{array}{l}\text { \% } \\
\text { difference } \\
\text { (group } 1 / 3 \text { ) }\end{array}$ \\
\hline \multicolumn{8}{|c|}{ Trabecular microarchitecture } \\
\hline $\mathrm{aBV} / \mathrm{TV}$ & $0.61(0.11)$ & $0.62(0.10)$ & $0.72(0.12)$ & 0.04 & 1.6 & 16.1 & 18.0 \\
\hline aTb.Th & $1.26(0.43)$ & $1.19(0.34)$ & $1.50(0.44)$ & 0.24 & 5.6 & 26.1 & 19.0 \\
\hline aTb.Sp & $0.80(0.10)$ & $0.80(0.13)$ & $0.71(0.13)$ & 0.173 & 0.0 & 11.3 & 11.3 \\
\hline aTb.N & $0.51(0.11)$ & $0.53(0.10)$ & $0.50(0.09)$ & 0.74 & 3.9 & 5.7 & 2.0 \\
\hline \multicolumn{8}{|l|}{ Statistical texture } \\
\hline \multicolumn{8}{|l|}{ Histogram } \\
\hline Mean & 2017 & 2031 & 2327 & $<0.001$ & 0.7 & 14.6 & 15.4 \\
\hline Variance & 829700 & 819400 & 791900 & 0.77 & 1.2 & 3.4 & 4.6 \\
\hline Skewness & 0.22 & 0.15 & 0.23 & 0.11 & 31.8 & 53.3 & 4.5 \\
\hline Kurtosis & -0.61 & -0.71 & -0.43 & $<0.001$ & 16.4 & $39.4 \dagger$ & 29.5 \\
\hline \multicolumn{8}{|l|}{ Gradient } \\
\hline Mean & 1.54 & 1.44 & 1.03 & $<0.001$ & 6.5 & $28.5^{\dagger}$ & $33.1+$ \\
\hline Variance & 0.95 & 0.90 & 0.54 & $<0.001$ & 5.3 & $40.0+$ & $43.2+$ \\
\hline Skewness & 0.41 & 0.35 & 0.21 & $<0.001$ & 14.6 & $40.0 \dagger$ & 48.8 \\
\hline Kurtosis & 0.23 & 0.17 & -0.13 & $<0.001$ & 26.1 & $176.5^{\dagger}$ & $156.5^{\dagger}$ \\
\hline $\begin{array}{l}\text { Number of non-zero } \\
\text { gradient }\end{array}$ & 0.86 & 0.84 & 0.75 & $<0.001$ & 2.3 & $10.7+$ & $12.8+$ \\
\hline \multicolumn{8}{|c|}{ Run-length matrix } \\
\hline $\begin{array}{l}\text { Short run length } \\
\text { emphasis }\end{array}$ & 0.90 & 0.89 & 0.86 & $<0.001$ & 1.1 & $3.4 \dagger$ & $4.4 \dagger$ \\
\hline $\begin{array}{l}\text { Long run length } \\
\text { emphasis }\end{array}$ & 1.53 & 1.61 & 1.84 & $<0.001$ & 5.2 & $14.3^{\dagger}$ & $20.3 \dagger$ \\
\hline $\begin{array}{l}\text { Run length non- } \\
\text { uniformity }\end{array}$ & 3770 & 4009 & 4582 & $<0.001$ & 6.3 & $14.3^{+}$ & $21.5^{\dagger}$ \\
\hline $\begin{array}{l}\text { Grey-level non- } \\
\text { uniformity }\end{array}$ & 315.2 & 353.9 & 428.9 & $<0.001$ & 12.3 & 21.2 & $36.1+$ \\
\hline $\begin{array}{l}\text { Fraction of image in } \\
\text { runs }\end{array}$ & 0.87 & 0.86 & 0.82 & $<0.001$ & 1.1 & $4.7+$ & $5.7+$ \\
\hline \multicolumn{8}{|c|}{ Grey-level co-occurrence matrix } \\
\hline $\begin{array}{l}\text { Angular second } \\
\text { moment }\end{array}$ & 0.0061 & 0.0072 & 0.0120 & $<0.001$ & 18.0 & $66.7^{\dagger}$ & $96.7^{\dagger}$ \\
\hline Contrast & 25.29 & 23.58 & 10.82 & $<0.001$ & 6.8 & $54.1 \dagger$ & $57.2+$ \\
\hline Correlation & 0.43 & 0.48 & 0.48 & 0.01 & 11.6 & 0.0 & 11.6 \\
\hline Entropy & 2.39 & 2.35 & 2.08 & $<0.001$ & 1.7 & $11.5^{\dagger}$ & $13.0+$ \\
\hline $\begin{array}{l}\text { Inverse difference } \\
\text { moment }\end{array}$ & 0.26 & 0.28 & 0.34 & $<0.001$ & 7.7 & $21.4+$ & $30.8+$ \\
\hline Sum of squares & 23.4 & 23.3 & 10.5 & $<0.001$ & 0.4 & $54.9+$ & $55.1^{\dagger}$ \\
\hline
\end{tabular}

*all values are mean (SD).

tdifference is significant at $p<0.0025$ level 
TABLE 3. Results of between group comparisons at the lateral tibial plateau. Parameters demonstrating significant differences between groups are highlighted in bold.

\begin{tabular}{|c|c|c|c|c|c|c|c|}
\hline Parameter & Group 1 * & Group 2 * & Group 3* & $\begin{array}{l}\mathrm{p} \text { (all } \\
\text { groups) }\end{array}$ & $\begin{array}{l}\text { \% } \\
\text { difference } \\
\text { (group } 1 / 2 \text { ) }\end{array}$ & $\begin{array}{l}\% \\
\text { difference } \\
\text { (group 2/3) }\end{array}$ & $\begin{array}{l}\text { \% } \\
\text { difference } \\
\text { (group 1/3) }\end{array}$ \\
\hline \multicolumn{8}{|c|}{ Trabecular microarchitecture } \\
\hline $\mathrm{aBV} / \mathrm{TV}$ & $0.44(0.12)$ & $0.43(0.13)$ & $0.32(0.17)$ & 0.12 & 2.3 & 25.6 & 27.3 \\
\hline aTb.Th & $0.89(0.22)$ & $0.84(0.23)$ & $0.73(0.20)$ & 0.256 & 5.6 & 13.1 & 18.0 \\
\hline aTb.Sp & $0.89(0.17)$ & $0.89(0.15)$ & $1.07(0.34)$ & 0.165 & 0.0 & 20.2 & 20.2 \\
\hline aTb.N & $0.50(0.10)$ & $0.52(0.11)$ & $0.43(0.19)$ & 0.358 & 4.0 & 17.3 & 14.0 \\
\hline \multicolumn{8}{|l|}{ Statistical texture } \\
\hline \multicolumn{8}{|l|}{ Histogram } \\
\hline Mean & 2403 & 2447 & 3304 & $<0.001$ & 1.8 & $35.0+$ & $37.5+$ \\
\hline Variance & 1129000 & 999900 & 1902000 & $<0.001$ & 11.4 & $90.2+$ & $68.5+$ \\
\hline Skewness & 0.40 & 0.32 & 0.36 & 0.1 & 20.0 & 12.5 & 10.0 \\
\hline Kurtosis & -0.16 & -0.20 & 0.02 & $<0.001$ & 25.0 & $110.0+$ & $112.5+$ \\
\hline \multicolumn{8}{|l|}{ Gradient } \\
\hline Mean & 1.78 & 1.67 & 1.40 & $<0.001$ & 6.2 & 16.2 & $21.3+$ \\
\hline Variance & 1.22 & 1.04 & 0.82 & 0.01 & 14.8 & 21.2 & 32.8 \\
\hline Skewness & 0.53 & 0.44 & 0.38 & $<0.001$ & 17.0 & 13.6 & $28.3^{+}$ \\
\hline Kurtosis & 0.55 & 0.36 & 0.41 & 0.05 & 34.5 & 13.9 & 25.5 \\
\hline $\begin{array}{l}\text { Number of non-zero } \\
\text { gradient }\end{array}$ & 0.89 & 0.88 & 0.84 & $<0.001$ & 1.1 & 4.5 & $5.6+$ \\
\hline \multicolumn{8}{|c|}{ Run-length matrix } \\
\hline $\begin{array}{l}\text { Short run length } \\
\text { emphasis }\end{array}$ & 0.92 & 0.91 & 0.90 & $<0.001$ & 1.1 & 1.1 & $2.2+$ \\
\hline $\begin{array}{l}\text { Long run length } \\
\text { emphasis }\end{array}$ & 1.40 & 1.44 & 1.53 & $<0.001$ & 2.9 & 6.3 & $9.3+$ \\
\hline $\begin{array}{l}\text { Run length non- } \\
\text { uniformity }\end{array}$ & 4229 & 4186 & 3819 & 0.05 & 1.0 & 8.8 & 9.7 \\
\hline $\begin{array}{l}\text { Grey-level non- } \\
\text { uniformity }\end{array}$ & 291.1 & 328.2 & 334.3 & 0.1 & 12.7 & 1.9 & 14.8 \\
\hline $\begin{array}{l}\text { Fraction of image in } \\
\text { runs }\end{array}$ & 0.89 & 0.89 & 0.87 & $<0.001$ & 0.0 & 2.2 & $2.2+$ \\
\hline \multicolumn{8}{|c|}{ Grey-level co-occurrence matrix } \\
\hline $\begin{array}{l}\text { Angular second } \\
\text { moment }\end{array}$ & 0.0044 & 0.0055 & 0.0069 & 0.01 & 25.0 & 25.5 & $56.8^{\dagger}$ \\
\hline Contrast & 34.44 & 29.97 & 21.59 & 0.01 & 13.0 & 28.0 & 37.3 \\
\hline Correlation & 0.46 & 0.43 & 0.49 & 0.05 & 6.5 & 14.0 & 6.5 \\
\hline Entropy & 2.54 & 2.47 & 2.35 & $<0.001$ & 2.8 & 4.9 & $7.5+$ \\
\hline $\begin{array}{l}\text { Inverse difference } \\
\text { moment }\end{array}$ & 0.23 & 0.24 & 0.27 & $<0.001$ & 4.3 & 12.5 & $17.4{ }^{\dagger}$ \\
\hline Sum of squares & 32.7 & 27.1 & 21.6 & 0.01 & 17.1 & 20.3 & 33.9 \\
\hline
\end{tabular}

*all values are mean (SD).

tdifference is significant at $p<0.0025$ level 
TABLE 4. Number of subjects classified correctly by linear discriminant functions created using trabecular microarchitecture and statistical texture parameters.

\begin{tabular}{lll}
\hline & Medial tibial plateau & Lateral tibial plateau \\
\hline Trabecular microarchitecture & $12 / 30(40 \%, 22-58)^{*}$ & $9 / 30(30 \%, 14-46)$ \\
Statistical texture & $16 / 30(53 \%, 35-71)$ & $14 / 30(47 \%, 29-65)$ \\
\hline
\end{tabular}

*Data in parentheses are (percentage accuracy, 95\% Cl) 


\section{FIGURE LEGENDS}

Figure 1. Example coronal gradient echo images of the medial tibial plateau of (a) a 26 year old male group 1 subject, (b) a 44 year old male group 2 subject and (c) a 67 year old male group 3 subject. ROI placement in the tibial subchondral bone is demonstrated (white void rectangles).

Figure 2. Examples of sTA parameter differences between groups: (a) medial tibial plateau (MT) of a 21 year old male group 1 subject with high gradient kurtosis (0.82), (b) MT of a 45 year old male group 2 subject with low gradient kurtosis (-1.72) corresponding to an area of homogeneous low signal within the MT subchondral bone. (c) MT of a 26 year old male group 1 subject with low grey-level non-uniformity (218), (d) MT of a 46 year old male group 2 subject with high grey-level non-uniformity (505), corresponding to increased subchondral trabecular discontinuity. 


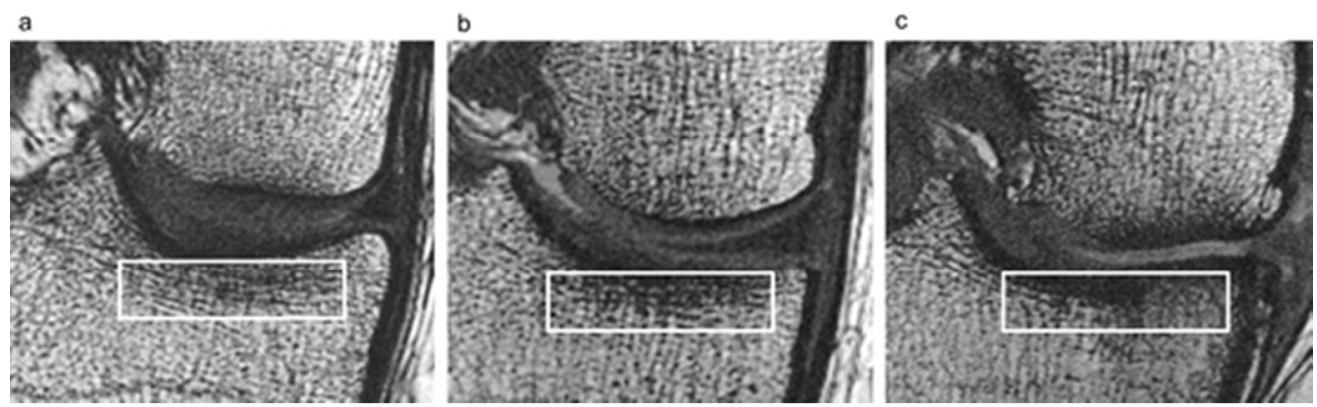

Example coronal gradient echo images of the medial tibial plateau of (a) a 26 year old male group 1 subject, (b) a 44 year old male group 2 subject and (c) a 67 year old male group 3 subject. ROI placement in the tibial subchondral bone is demonstrated (white void rectangles).

$$
46 \times 14 \mathrm{~mm}(300 \times 300 \text { DPI) }
$$


a

b
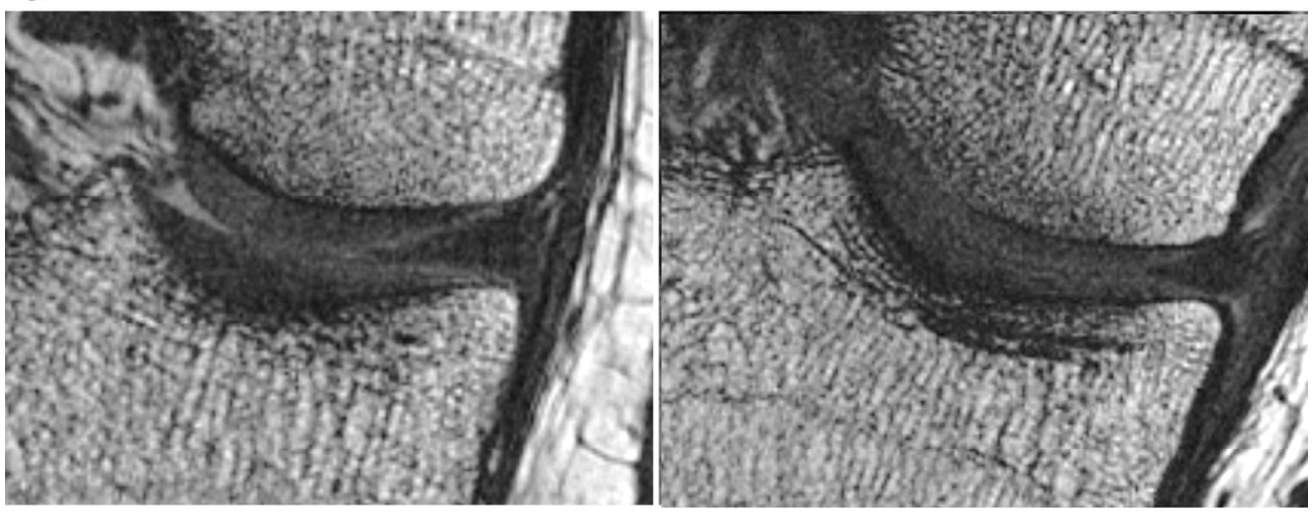

C

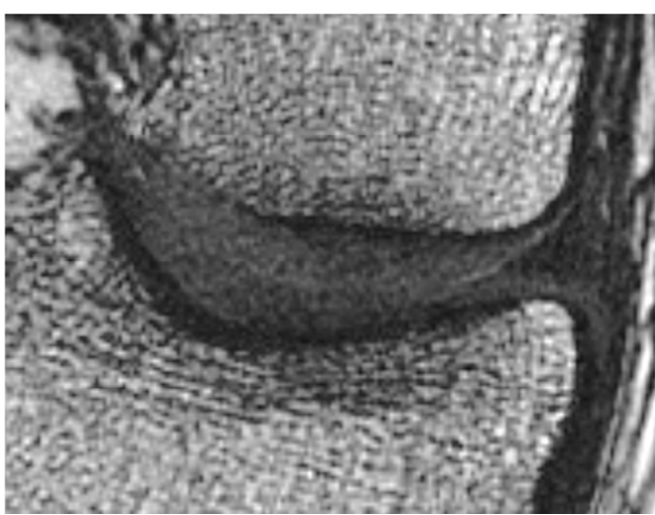

d

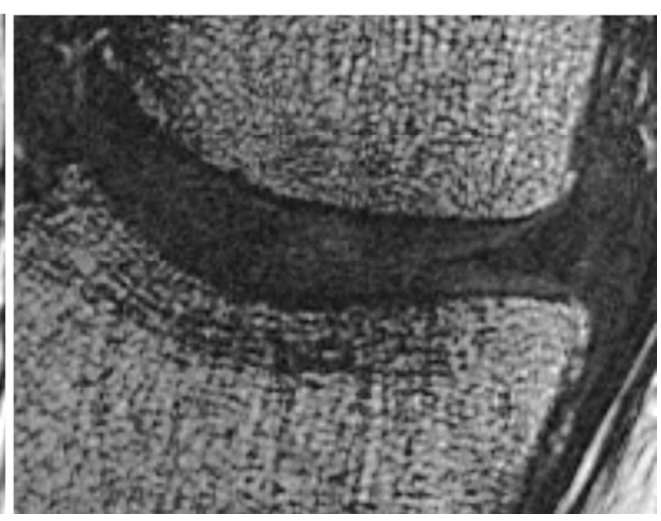

Examples of sTA parameter differences between groups: (a) medial tibial plateau (MT) of a 21 year old male group 1 subject with high gradient kurtosis (0.82), (b) MT of a 45 year old male group 2 subject with low gradient kurtosis $(-1.72)$ corresponding to an area of homogeneous low signal within the MT subchondral bone. (c) MT of a 26 year old male group 1 subject with low grey-level non-uniformity (218), (d) MT of a 46 year old male group 2 subject with high grey-level non-uniformity (505), corresponding to increased subchondral trabecular discontinuity. $111 \times 97 \mathrm{~mm}(300 \times 300$ DPI $)$ 